first is possible and the second impossible but that these facts are not known a priori. In this paper, criteria are worked out for ascertaining a priori whether a solution is impossible when $A<(B+C)$. Incidentally, some properties of cases in which $A=B+C$ are noted.

R. G. D. Richardson, Secretary.

\title{
THE OCTOBER MEETING OF THE SAN FRANCISCO SECTION
}

The fortieth regular meeting of the San Francisco Section of the American Mathematical Society was held at the University of California, October 21, 1922. Professor Allardice presided during the early part of the meeting and Professor Cajori during the latter part. The total attendance was thirty-five, including the following twenty-four members of the Society:

Alderton, Allardice, Andrews, Barter, Bernstein, Blichfeldt, Buck, Cajori, Edwards, Growe, Haskell, Hoskins, Irwin, Lehmer, Levy, Libby, Moreno, F. R. Morris, T. M. Putnam, Shane, P. Sperry, Stromquist, A. R. Williams, Wong.

The following officers were chosen for the year: Chairman, Professor Florian Cajori; Secretary, Professor B. A. Bernstein; Programme Committee, Professors H. F. Blichfeldt, D. N. Lehmer, B. A. Bernstein.

It was decided to hold the next Fall meeting of the Section on Saturday, October 20, 1923, at the University of California.

Titles and abstracts of papers read at this meeting follow. The papers of Professors Bell and Smail were read by title.

1. Dr. J. D. Barter: Spiral functions. Preliminary report.

If $\alpha$ is a root of the equation $a_{0}+a_{1} x+a_{2} x^{2}+\cdots$ $+\lambda+a_{n} x^{n}=0$, termed the "fundamental equation," then exp. $(\alpha t)=f_{0}(t)+\alpha f_{1}(t)+\alpha^{2} f_{2}(t)+\cdots+\lambda+\alpha^{n-1} f_{n-1}(t)$. The functions $f$ are termed spiral functions. Their general properties and applications are summarized. Methods for calculating the values of these functions in special cases are outlined. 
2. Professor E. T. Bell: Applications of analysis to the arithmetic of higher forms.

In papers published in the Transactions of this Society, it has been shown that any identity between elliptic, abelian or theta functions gives rise to a relation between functions wholly arbitrary except as to parity, the arguments of the functions being linear functions of the indeterminates representing an arbitrary integer in a quadratic form in several variables. In the present paper the restriction that the forms be of the second degree is removed. The method of paraphrase is thus widely generalized.

3. Professor E. T. Bell: Umbral symmetric functions and algebraic analogues of the Bernoullian and Eulerian numbers and functions.

The numbers of Bernoulli and Euler of rank $n$ are replaced by rational integral algebraic functions in $n$ independent variables. For unit values of all the variables the functions degenerate to the numbers of the same rank; the general relations between the numbers are special cases of relations between the functions. This paper is complementary to another, to be published in the Transactions of THIs Society, in which the numbers of rank $n$ are replaced by polynomials of degree $n$ in one variable.

4. Professor B. A. Bernstein: An arithmetic representation of Boolean logic.

The author shows how Boolean logic can be represented by arithmetic operations. The complete paper will appear in a later number of this Bulletin.

5. Professor B. A. Bernstein: Arithmetic independence systems for the Whitehead-Huntington postulates for Boolean algebras.

The author exhibits simple arithmetic systems which may take the place of the abstract systems used by Huntington in proving the independence of his first set of postulates for Boolean algebras. The complete paper will appear in a later nnmber of this BuLLETIN. 
6. Professor B. A. Bernstein: A Boolean representation of a number field.

In this paper the author gives a representation of a twoelement field by means of operations of a Boolean algebra, and adds a note on the existence of abelian groups in any Boolean algebra. The complete paper will appear in a later number of this BulletiN.

7. Professor Florian Cajori: Origin of the symbols for "degrees, minutes, and seconds."

The signs ${ }^{\circ}$, , $^{\prime \prime}$ originated in the sixteenth century in the use of the exponential concept to operations involving sexagesimal numbers. The sign ${ }^{\circ}$ has been traced back to $\mathrm{J}$. Peletier (1558).

8. Professor Florian Cajori: The St. Andrew's Cross $\times$ as a mathematical symbol.

The claim made by certain writers that $X$ was adopted as the sign of multiplication on the European continent before the English adopted it in 1618 is found to be invalid. Before 1618 other uses (ten different ones) were made of two obliquely intersecting lines. This paper will appear in the MatheMATICAL GazetTE.

9. Professor Florian Cajori: Origin of the names arithmetical and geometrical progression and proportion.

Though not used by Euclid, Apollonius and Archimedes, these names originated earlier, in the time of Archytas and Hippasus; they met with more common adoption 400 years after Euclid. Aristotle uses the term "geometric proportion." The names indicate the fields of Greek mathematics in which the respective proportions found their widest application. This paper will be published in School Science and MatheMATICS.

10. Professor L. L. Smail: Applications of vector analysis to celestial mechanics. Preliminary report.

The author has applied the methods of vector analysis to the problem of two bodies and to the discussion of the general integrals of the problem of $n$ bodies, and has obtained some simplifications of treatment over the usual methods. 
11. Dr. A. R. Williams: Note on the form of a space curve in the vicinity of a singular point. Preliminary report.

Von Staudt noted that the projections of a space curve on the three principal planes could be deduced from simple geometrical considerations. Christian Wiener prepared models illustrating the eight possible cases. The analytic side of the question has been treated at some length by several writers. The author shows that a simple analysis can be made by means of the torsion function.

12. Professor L. M. Hoskins: Note concerning estimates of the rigidity of the earth.

Estimates of the rigidity of the earth are based upon two independent lines of observational evidence, one showing the effect of tidal forces in changing the direction of apparent gravity, the other the prolongation of the earth's period of free nutation attributed to changes in centrifugal forces as the axis of rotation changes. Estimates based upon the two kinds of evidence have not been in close agreement. The author has shown (Transactions of this Society, vol. 21) that the two kinds of evidence may be harmonized by perfecting the theory of strain of an elastic sphere so as to take account of variation of density and elastic moduli. In the computations leading to this conclusion, however, a law of variation of density was for simplicity used which involved a considerable error in the value of the surface density as well as an error of about 3 per cent in the moment of inertia. Computations have now been made with a density formula which corrects these discrepancies and causes no important change in the quantities upon which estimates of rigidity are based. The formula used agrees rather closely with Laplace's law of density.

B. A. Bernstein, Secretary of the Section. 